\title{
EN TORNO A LA LIBERTAD, LA IGUALDAD Y LA SEGURIDAD COMO DERECHOS HUMANOS BÁSICOS (Acotaciones a Liborio Hierro)
}

\author{
Ricardo García Manrique \\ ESADE-Universitat Ramon Llull (Barcelona)
}

\section{Sentido de la contraponencia}

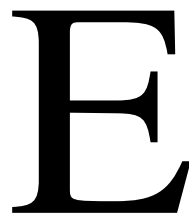

1 texto de Liborio Hierro que precede a éste constituye una aguda reflexión sobre los aspectos centrales de una teoría moral de los derechos humanos y, sin duda, una atractiva respuesta a la pregunta que vertebraba el seminario en el que se presentó como ponencia: ¿Cuál es el «núcleo duro» de los derechos humanos? Mi propósito consiste simplemente en formular una serie de acotaciones con el deseo de que ayuden a iluminar un poco más algunas de las cuestiones que se propone resolver. De este modo prolongaré la función de contraponente que desempeñé en aquel seminario, celebrado en abril de 1999. El trabajo de Liborio Hierro tiene un alcance muy amplio, por lo que renuncio de antemano a comentarlo de forma sistemática e integral. A cambio, he seleccionado algunas de las tesis que propone, las que me parecen más interesantes, sobre las cuales versarán mis observaciones. Esas tesis contienen:

(1) Una forma de plantear la pregunta acerca de cuál es el núcleo duro de los derechos humanos.

(2) Una definición de los derechos humanos.

(3) La identificación de los tres derechos humanos más importantes, la determinación del contenido de cada uno de ellos y la fijación de unas reglas que permitan ordenarlos entre sí.

\section{La forma de plantear la pregunta}

La forma en que Liborio Hierro aborda la pregunta del seminario me parece acertada por dos razones: en primer lugar, porque, en efecto, encierra dos cuestiones diferentes, a saber: ¿cuál es el pedigrí de los derechos humanos? y ¿qué jerarquía existe entre éstos?; en segundo lugar, porque muy probablemente sea cierto que la respuesta a la segunda pregunta depende de la respuesta a la primera, de modo que parece que ambas forman parte de un único argumento. «Núcleo duro», en el primer caso, significa «esencia» 
de cada uno de los derechos, aquéllo que está presente en todos los derechos humanos y no en el resto de los derechos, noción a través de la cual, por tanto, podemos distinguir a unos de otros. En el segundo caso, «núcleo duro» significa «esencia» del conjunto de los derechos humanos; en este caso, se trata de determinar qué derechos humanos son especialmente relevantes (esenciales) por alguna razón. La diferencia entre una y otra pregunta se aprecia muy bien en el contenido de la respuesta: en el primer caso, el núcleo duro será algún carácter presente en los derechos humanos; en el segundo caso, el núcleo duro será un grupo de derechos humanos. Pues bien, parece que sólo indagando en la esencia de los derechos humanos podremos determinar cuáles son los derechos humanos esenciales.

Sin embargo, es posible que, aun determinando correctamente ambas preguntas y la relación entre ellas, no sea posible responder a alguna de las dos. En mi opinión, esto es lo que sucede, que es posible responder a la primera, pero no a la segunda. En síntesis, creo que es correcto determinar la esencia de los derechos humanos a partir de la idea de autonomía moral, pero me parece que ello no permite identificar un grupo de derechos humanos como derechos humanos «esenciales», puesto que todos ellos constituyen un conjunto indivisible de técnicas al servicio de dicha autonomía moral.

\section{La definición de los derechos humanos}

Liborio Hierro propone la siguiente definición de los derechos humanos, de carácter estipulativo:

Los derechos humanos son aquellas libertades, inmunidades, pretensiones y potestades que corresponden a todo ser humano como condición necesaria para realizarse como sujeto moral y cuya satisfacción es condición necesaria y suficiente para justificar la existencia, el origen y el contenido de un sistema jurídico.

Esta definición contiene un elemento formal y un elemento material. El elemento formal define la estructura normativa de los derechos («libertades, inmunidades, pretensiones y potestades») y el elemento material define el contenido de los derechos, en este caso por remisión a la vinculación con la realización de los individuos como sujetos morales y a la justificación de un sistema jurídico ${ }^{1}$. De esta definición quiero destacar tres aspectos encadena-

\footnotetext{
${ }^{1}$ De acuerdo con Luis Prieto, un concepto «funcional» $\mathrm{o}$ «formal» de los derechos no permite determinar cuáles son sino cómo son: «los derechos no son éstos o aquéllos, sino el soporte o recipiente institucional que pretende traducir en requerimientos jurídicos esas exigencias morales importantes»; un concepto «material», por el contrario, se pregunta por cuáles son esos derechos, o bien qué elementos debe reunir una pretensión o un derecho subjetivo para convertirse en derecho humano (Prieto 1994, 183). La definición de Liborio Hierro, aunque contenga un elemento formal, da lugar a un concepto material de derechos humanos, porque el elemento formal se limita a incluirlos en la categoría más amplia de los derechos subjetivos, pero el elemento definidor específico de los derechos humanos no es formal sino material.
} 
dos entre sí. En primer lugar, los derechos humanos son definidos como una clase de los derechos subjetivos (y, más concretamente, como una clase de los derechos subjetivos morales). Esto implica que no podrán incluirse entre ellos ciertos objetivos sociales que no puedan configurarse bajo la forma del derecho subjetivo, lo cual tiene alguna consecuencia importante que indico a continuación. En segundo lugar, los derechos humanos son definidos como «condición necesaria» de la realización moral de los individuos. Liborio Hierro da cuenta en la nota 12 de una observación que le hice: los derechos humanos no pueden ser condición necesaria y suficiente de la realización moral de los individuos; pero no sólo, como él indica, «porque han de darse otras condiciones extranormativas como, por ejemplo, ciertos niveles mínimos de salud física y mental», sino también porque creo que no todas las condiciones normativas requeridas para la realización moral pueden revestir la forma de derechos subjetivos (estoy pensando en objetivos sociales tales como la conservación del medio ambiente, la promoción de la cultura o la destrucción de las armas nucleares). En tercer lugar, la satisfacción de los derechos humanos es considerada «condición necesaria y suficiente para justificar la existencia, el origen y el contenido de un sistema jurídico»; pues bien, la observación inmediatamente anterior permite poner en cuestión la condición suficiente: un sistema jurídico no se justificaría sólo por garantizar la satisfacción de los derechos humanos, sino que tendría que garantizar también la satisfacción de otros objetivos sociales ${ }^{2}$.

\section{La elección de los derechos básicos}

Liborio Hierro propone la libertad, la igualdad y la seguridad como «candidatos a una nómina completa de derechos humanos». En sentido estricto, no se trata, por tanto, de la determinación de un «núcleo» de derechos humanos básicos, sino de un catálogo cerrado de derechos humanos. Las tres nociones (libertad, igualdad y seguridad) aparecen en la mayoría de teorías de los derechos humanos, pero no siempre como derechos. El propio autor cita a Eusebio Fernández, pero éste no «reduce los derechos humanos básicamente a tres valores, principios o criterios», sino que entiende que los derechos humanos se fundamentan en estos tres valores, en el sentido de que son mecanismos jurídicos que sirven a su realización ${ }^{3}$. La diferencia, al

\footnotetext{
2 Además, la justificación de un sistema jurídico requeriría, en todo caso, razonar la necesidad de recurrir a la coacción para lograr los fines propuestos, puesto que, cuando menos, cabe imaginar la posibilidad de que esos fines pudieran alcanzarse sin recurrir a una técnica intrínsecamente negativa desde un punto de vista moral como es la coacción; justificar un sistema jurídico requiere examinar dicha posibilidad y rechazarla. Liborio Hierro no se ocupa de esta cuestión, así que yo tampoco lo haré.

${ }^{3}$ Fernández 1984, 120. Similar es la teoría de los derechos fundamentales de Gregorio Peces-Barba, en el sentido de que distingue valores y derechos (Peces-Barba 1995, 209).
} 
menos en principio, parece importante: una cosa es afirmar que de la autonomía moral individual se deducen tres valores que constituyen la carga moral completa de una teoría de los derechos humanos ${ }^{4}$; otra cosa distinta es asumir que dichos valores son en realidad derechos humanos en sentido estricto, y que no hay más derechos humanos que los tres indicados. El significado de la expresión en cursiva debe ser aclarado, porque el propio Hierro se refiere más adelante a otros derechos, e incluso afirma que la nómina de derechos no puede cerrarse definitivamente. Habrá que entender, por tanto, que existen dos categorías de derechos, pero no en el sentido de que unos sean distintos de otros (aunque más importantes), sino en el sentido de que unos incluyen a los otros: libertad, igualdad y seguridad incluirían al resto de los derechos, que serían especificaciones de estos tres (derechos «derivados», si se me permite la expresión). Así, por tanto, habría que modificar la primera impresión y reconocer que, en efecto, Liborio Hierro sí define un núcleo duro de derechos humanos, integrado por tres de sus miembros.

Ahora bien, si se establecen dos categorías de derechos humanos, creo que sería necesario aclarar en qué se diferencian unos y otros derechos, si esta diferencia se refiere sólo a su alcance moral o si también afecta a la forma de su articulación jurídica, por ejemplo. También, dado que los derechos humanos básicos son considerados por el autor como derechos prima facie, si esta consideración se extiende también a los derechos humanos derivados, y en qué aplicaciones técnico-jurídicas se traduce. En tercer lugar, Hierro afirma que no es posible cerrar la nómina de los derechos morales básicos, dado que «las necesidades del sujeto para desenvolverse como agente moral son necesidades relativas al contexto»; ¿se refiere sólo a los derechos derivados o también a la nómina constituida por la libertad, la igualdad y la seguridad? En definitiva, lo que me estoy preguntando es qué consecuencias concretas se deducen del hecho de considerar a la libertad, la igualdad y la seguridad como auténticos derechos subjetivos y no sólo como valores, teniendo en cuenta además la existencia de otra categoría de derechos (los derechos derivados). Creo que Liborio Hierro debería precisar más el alcance de esta distinta calificación.

En cualquier caso, hay que señalar que la libertad, la igualdad y la seguridad no pueden identificar un núcleo duro de derechos humanos si no son definidas en términos más precisos. Es difícil imaginar una teoría de los derechos humanos que estuviera dispuesta a rechazar que alguna de las tres

${ }^{4}$ Para saber si esa carga moral es, en efecto, completa, habría que discutir si la solidaridad debería figurar al lado de la libertad, la igualdad y la seguridad (cuestión aludida pero no desarrollada por Liborio Hierro), tal como propone Peces-Barba (Peces-Barba 1995, 213 y 261-282, y Peces-Barba 1993, 294-318). Sobre el estatuto normativo de la solidaridad, véanse también González Amuchastegui 1991 y De Lucas 1993. 
formen parte de su contenido, puesto que hay una conexión muy próxima entre cada una de ellas y alguno de los presupuestos de todo ese grupo de teorías: la libertad es correlato de la autonomía individual que se predica como fundamento de los derechos; la igualdad deriva del dato fáctico de la semejanza de todos los seres humanos que toda teoría de los derechos acepta (con algunos matices a que después haré alusión); la seguridad es un elemento lógico de todo sistema normativo, y lo que trata de favorecer cualquier teoría de los derechos es precisamente el establecimiento de un sistema normativo. Por tanto, un núcleo con el que estarían de acuerdo desde Hobbes hasta Rawls no puede ser muy duro, sino más bien blando. La dureza habrá que ir a buscarla en la definición precisa del contenido de cada uno de los tres derechos, donde se encontrará lo que de específico pueda tener la propuesta de Liborio Hierro.

\section{El derecho a la libertad}

Liborio Hierro se ocupa de tres cuestiones en relación con la libertad: «si existe o no un derecho general a la libertad, cuáles son y cómo se relacionan las formas de la libertad y el carácter cualitativo de la libertad». Comentaré alguna cosa respecto de las dos primeras.

El derecho general de libertad. La opinión de Hierro es que, en contra de la tesis de Dworkin, sí existe un derecho general a la libertad. Lo que no sé es si Hierro piensa en ese derecho del mismo modo en que lo hace Dworkin. La divergencia radica, creo, en la distinta forma de usar el término «derecho». Para Hierro, el derecho general de libertad consiste en hacer todo lo que las normas no prohíben expresamente, y nada más, puesto que, dado el carácter cualitativo de la libertad, que Hierro asume más adelante, ese derecho general cede ante «razones necesarias de coordinación social». Para Dworkin, un derecho es una posición normativa especialmente fuerte, al menos tanto como para no tener que ceder ante razones necesarias de coordinación social, y el derecho general a la libertad no constituye una posición normativa tal ${ }^{5}$. Por lo tanto, el desacuerdo entre Hierro y Dworkin no afecta al contenido del derecho general de libertad, sino al uso de la categoría «derecho» para esa libertad general, que Hierro acepta y Dworkin rechaza.

También cabe considerar la posibilidad de que esa libertad general, tal como Hierro la define, más que un derecho, sea esa regla lógica propia de los sistemas normativos que dice: «todo lo no prohibido está permitido», re-

${ }^{5}$ Dworkin 1984, 380-388. Dworkin escribe expresamente (pág. 384): «Si alguien tiene derecho a algo, está mal que el gobierno se lo niegue, aunque negárselo favoreciera el interés general», y además: «No creo que el derecho a la libertad significara mucho (...) si se apoyara en algún sentido más débil que el citado». 
gla que, por cierto, bien podría formularse al revés («todo lo no permitido está prohibido»); en este caso, según Hierro, «se estaría negando la autonomía como carácter moral originario de los sujetos», pero puede ser que eso dependa de los términos en que se definan las prohibiciones y los permisos respectivamente, de manera que quizá un sistema normativo que acepte la primera norma general pueda restringir más la autonomía que uno que acepte la segunda. Esto sucedería si las prohibiciones del primer sistema se definieran en términos muy amplios (quedando poco margen para la actuación de la regla general de permiso) o si los permisos del segundo sistema se definieran en términos muy amplios (quedando poco margen para la actuación de la regla general de prohibición). Lo que estoy planteando es la posibilidad de que esta cuestión tenga un carácter más lógico que moral.

Las formas de la libertad. La tesis de Hierro en este punto es aceptar que las formas de la libertad son dos: la libertad positiva y la libertad negativa, y en insistir, en contra de Berlin, en que ambas están relacionadas, además de rechazar la noción de libertad real o material, idea que califica como «poco afortunada» y «errónea». En cuanto a la vinculación entre libertad negativa y positiva, creo que lleva razón Hierro cuando afirma que «ambas consisten en el ejercicio de la autonomía, si bien en diferentes contextos», definidos por la posibilidad de que ese ejercicio se pueda desarrollar individualmente (libertad negativa) o tenga que serlo colectivamente (libertad positiva); «la libertad positiva -concluye Hierro- no es, por tanto, otra cosa que el ejercicio de la autonomía personal en condiciones de interdependencia». La relevancia de esta conexión radica en que impide la opción por una de las dos libertades en detrimento de la otra, dado que ambas sirven a un mismo objetivo: la maximización de la autonomía. No cabría, por tanto, preferir la libertad negativa a la libertad positiva si se admite que ambas son instrumentos necesarios para el desarrollo de la autonomía individual.

En cuanto a la libertad real o material, Hierro rechaza que constituya una modalidad de la libertad, puesto que «una cosa es la libertad y otra las condiciones para ejercerla». Siendo así, me pregunto cómo aplicar estas categorías en un ejemplo como el que sigue. Supongamos que en un país determinado sólo existen escuelas privadas. $X$ e $y$ son dos individuos en edad escolar y residentes en dicho país. La familia de $x$ tiene el dinero suficiente para pagar el precio de una escuela privada y la familia de $y$ no lo tiene. Por tanto, el primero puede educarse y el segundo, no. ¿Qué deberemos decir? Según lo visto, que ambos tienen la libertad para educarse y que sólo $x$ reune las condiciones para ejercerla. Sin embargo, yo me pregunto si $y$ tiene en verdad dicha libertad en el mismo sentido en que la tiene $x$. Si $y$ se acerca a la escuela, le será vedado el paso, puesto que la escuela tiene el derecho para ello, en virtud de una norma que dice algo así: «toda escuela podrá vedar 
el acceso a todos aquellos que no aporten el dinero necesario para pagar la matrícula establecida». Este impedimento es, evidentemente, jurídico, puesto que ha sido establecido por una norma jurídica (del mismo modo que la propiedad de bienes no es una situación natural, sino el resultado de la aplicación de normas jurídicas), y se parece mucho al que establece una norma como ésta: «toda escuela podrá rechazar el acceso a las niñas». Cabría alegar que las niñas no han elegido serlo (alegar que el sexo forma parte de la «identidad no negociable»), pero parece que los niños pobres tampoco han elegido la pobreza, y en determinados contextos dejar de ser pobre parece tan difícil o más que dejar de ser mujer. Por tanto, si en el caso de la discriminación sexual decimos que no existe libertad de educación para las niñas (y no que estas personas «no reúnen las condiciones para ejercer la libertad»), no veo por qué no hemos de decirlo en el caso de la discriminación económica. De aquí deduzco que no está tan claro que el concepto de libertad real o material sea tan desafortunado, si al menos sirve para poner de manifiesto un cierto tipo de discriminaciones en el disfrute de la libertad; soy consciente de que se me puede acusar de no haber comprendido la distinción entre libertad y capacidad trazada por Berlin, Oppenheim, Hayek o Aron, pero precisamente esa distinción es la que no veo tan clara ${ }^{6}$.

\section{El derecho a la igualdad}

El derecho a la igualdad, tal como lo establece Liborio Hierro, reviste dos formas: la igualdad formal o igualdad ante la ley, y la igualdad material, o igualdad de oportunidades. Me pregunto si es necesario configurar a la igualdad como derecho (o como valor) para formular una teoría de los derechos humanos, esto es, me pregunto si la igualdad tiene un estatuto axiológico equivalente al de la libertad. Con el fin de proponer una respuesta, supondré tres premisas que creo necesarias para cualquier teoría de los derechos humanos, que permiten calificarla como una teoría igualitaria:

(1) Una teoría de los derechos humanos es una teoría normativa (en el sentido de que su objetivo consiste en proponer normas).

(2) Una teoría de los derechos humanos es una teoría de la justicia.

(3) Los seres humanos son básicamente iguales, en tanto igualmente capaces para la autodeterminación moral.

Cada una de las tres premisas contiene una referencia a la igualdad que permite afirmar, en tres sentidos, que la teoría de los derechos humanos es igualitaria. En la primera, la igualdad aparece como elemento lógico de la idea de norma. Toda norma es un esquema de interpretación de la realidad ${ }^{7}$

\footnotetext{
${ }^{6}$ Véase la exposición y defensa de la distinción en Laporta 1983, 29-31.

${ }^{7}$ Cf., por ejemplo, Ross 1997, 34-42.
} 
que permite tratar conjuntamente una serie de casos, extrayendo de todos ellos un elemento común, esto es, suponiendo que, en algún aspecto, todos ellos son iguales. La aplicación de normas es, por tanto, una conducta regular o, en otros términos, exige tratar igual los casos iguales (de acuerdo con el criterio de igualdad necesariamente presente en toda norma). En este sentido, la igualdad es una idea necesaria para entender la idea de norma y, por eso, en tanto teoría que se propone formular normas, la teoría de los derechos humanos es una teoría igualitaria.

En la segunda premisa, la igualdad aparece en tanto elemento constitutivo de la justicia («médula de la justicia», la denomina $\operatorname{Radbruch}^{8}$ ). La idea de justicia no es una idea que forme parte de una teoría de los derechos humanos; más bien, una teoría de los derechos humanos es una teoría de la justicia, esto es, una teoría que responde a las dos preguntas que es necesario resolver para actuar justamente. Recordemos el concepto clásico de justicia: justicia es tratar igual a los iguales y desigual a los desiguales ${ }^{9}$. Pues bien: hace falta saber, primero, en función de qué son iguales o desiguales las personas, y, segundo, qué tratamiento merecen. Sólo actuaremos de acuerdo con la justicia si antes respondemos correctamente a estas dos preguntas, y una teoría de los derechos humanos no es sino un intento de definir el contenido de las dos respuestas ${ }^{10}$. A la primera pregunta, responde: las personas son iguales en su dignidad y, por tanto, tienen que ser tratadas igualmente en función de dicha dignidad; a la segunda pregunta responde: todas las personas merecen la titularidad de un cierto conjunto de derechos (los derechos humanos). El caso es que, en tanto teoría de la justicia, la teoría de los derechos humanos es, en el sentido indicado, una teoría necesariamente igualitaria.

En la tercera premisa, la igualdad aparece como un dato de hecho: todos los seres humanos son iguales porque son igualmente capaces para la autodeterminación moral. Rawls, por ejemplo, lo expresa con estas palabras:

Son precisamente las personas morales las que tienen derecho a una justicia igual. Las personas morales se distinguen por dos características: la primera, que son capaces de tener (y se supone que de adquirir) un sentido de su bien (expresado por un proyecto racional de vida); y segunda, que son capaces de tener

${ }^{8}$ Radbruch 1974, 31.

${ }^{9}$ El concepto se encuentra ya en Aristóteles (1985, 243-245 y 1989, 83); la fórmula del texto es de Radbruch (1974, 33), resumiendo la que incluye en su Política el propio Aristóteles: «Parece que la justicia consiste en la igualdad, y así es, pero no para todos, sino para los iguales, y la desigualdad parece ser justa, y lo es, en efecto, pero no para todos, sino para los desiguales» $(1989,83)$.

${ }^{10}$ No obstante, Radbruch cree que esas respuestas ya no forman parte de la idea de la justicia (que, por esa razón, es una idea formal), sino de la idea de «adecuación a fin» (Radbruch 1974, 34-35). 
(y se supone que de adquirir) un sentido de la justicia (...) Doy por supuesto que la capacidad de un sentido de la justicia es poseída por la abrumadora mayoría de la humanidad (...) No hay raza ni grupo reconocido de seres humanos que carezca de este atributo. Solamente los individuos dispersos no gozan de esta capacidad, o de su realización en un grado mínimo, y la imposibilidad de realizarla es la consecuencia de circunstancias sociales injustas o deterioradas, o de contingencias fortuitas ${ }^{11}$.

Obsérvese que esta igualdad debe entenderse como un hecho, independientemente de que resulte valiosa la circunstancia respecto de la cual los seres humanos son iguales; es decir, todos somos iguales en cuanto capaces de desarrollar una actitud moral, y eso, en sí, no es bueno ni malo, sino un hecho ${ }^{12}$. Una teoría de los derechos humanos es, por tanto, igualitaria en este tercer sentido.

Tenemos, pues, tres apariciones de la igualdad: la primera es una igualdad lógica, la tercera es una igualdad fáctica, y sólo la segunda es una igualdad axiológica, pero incluso en este caso parece más conveniente reservar el carácter axiológico para la justicia. La cuestión es saber si una teoría de los derechos humanos requiere, además de estas tres igualdades, alguna más, esto es, si requiere configurar la igualdad como valor o como derecho autónomo. Para ello, examinaré la igualdad ante la ley y la igualdad de oportunidades, que son los dos candidatos propuestos por Liborio Hierro para definir el derecho a la igualdad, con el fin de determinar si pueden ser consideradas como derivaciones lógicas de las tres premisas avanzadas.

La igualdad ante la ley puede significar dos cosas: en sentido amplio, consiste en que las normas (las leyes) sean aplicadas igualmente a todos los sujetos, sin tener en cuenta otras circunstancias que las establecidas por la propia norma; en este sentido amplio, parece que la igualdad ante la ley no es otra cosa que la igualdad lógica contenida en la premisa (1). Kelsen escribe, en este sentido:

No hay que confundir el principio de igualdad, postulado por la autoridad que crea la ley con la intención de hacerla igualitaria, con el principio de igual-

${ }^{11}$ Rawls 1985, 557-559. Véase, en general, todo el epígrafe 77, «La base de la igualdad». Otros, sin embargo, consideran que «la posesión de una personalidad moral no constituye una base satisfactoria para el principio de que todos los seres humanos son iguales» (Singer 1991, 30), por dos razones: porque la posesión de personalidad moral es una cuestión de grado y porque algunos seres humanos no la tienen en absoluto (los niños y ciertos enfermos). Singer, propone, en cambio, el principio de igual consideración de los intereses como base de la igualdad humana.

${ }_{12}$ En contra de la consideración fáctica de la igualdad en este contexto, véase De Lucas 1996, 493-494 («La igualdad no puede ser sino un constructo, un artificio. La naturaleza (...) no nos ha hecho iguales (...) Por eso, decir igualdad exige otro punto de vista que el descriptivo (...) La igualdad sólo puede ser admitida como concepto normativo, como idea, como respuesta»). De Lucas da la sensación de estar más cerca de Singer que de Rawls en este punto. 
dad ante la ley, destinado a las autoridades que aplican la ley a casos concretos. Los órganos que aplican la ley, al decidir un caso, no establecen ninguna diferencia que no se encuentre en la ley que se va a aplicar, es decir, aplican la ley tal como se debe hacer según su significado. Éste es el principio de legalidad, de la legitimidad inmanente a cualquier orden legal. A veces se presenta este principio como justicia de la ley, pero en realidad no tiene nada que ver con la Justicia $^{13}$.

(1) ${ }^{14}$ En sentido estricto, la igualdad ante la ley tiene un sentido histórico muy concreto, como Bobbio ha puesto de relieve ${ }^{15}$. Surge como respuesta a la discriminación estamental propia del Antiguo Régimen, y consiste en afirmar que las personas no pueden ser tratadas desigualmente en función del estamento al que pertenecen (ni, tampoco, ulteriormente, en función de su raza, de su sexo, etc.); esto es, la igualdad ante la ley no es más que otra forma de expresar que los seres humanos no son desiguales en función de ciertas circunstancias que, hasta un momento histórico determinado, sí se consideraban relevantes. El contenido concreto actual de la igualdad ante la ley es el resultado de considerar que determinadas circunstancias son irrelevantes para la creación y aplicación de normas, mientras que otras sí lo son (por ejemplo, la posesión de una nacionalidad determinada). En este segundo sentido, creo que la igualdad ante la ley es una conclusión lógica de las premisas (2) y (3), dado que si los individuos no se diferencian de manera relevante por su raza, sexo, creencias, etc.,no sería justo que estas circunstancias fueran tenidas en cuenta en la elaboración de las normas ${ }^{16}$.

En cuanto a la igualdad material, entendida como igualdad de oportunidades, cabe razonar así: si las personas merecen un determinado tratamiento precisamente en función de aquéllo en que son iguales (su autonomía moral, o su capacidad para desarrollarla), entonces no hay motivo para discriminarlas en dicho tratamiento y sí para tratarlas igualmente; afirmar esto no requiere añadir una nueva forma de igualdad, sino simplemente reflexionar sobre lo que las personas merecen en función de lo que tienen en co-

${ }_{13}$ Kelsen 1991, 51. Véanse también Sadurski 1986, 132; Laporta 1985, 6-7; Peces-Barba 1995, 284-285; y Hierro 1998, 104. En defensa del valor axiológico de la igualdad ante la ley, véase Ruiz Miguel 1997.

${ }^{14} \mathrm{Si}$ no lo interpreto mal, el propio Liborio Hierro sostiene esta tesis (Hierro 1998, 104); también Laporta (1985, 6-7) y, con matices, Peces-Barba (1995, 284-285).

15 Bobbio 1993, 72; también De Lucas 1996, 496.

${ }^{16}$ El Tribunal Constitucional español, a la hora de interpretar la igualdad ante la ley establecida por el artículo 14 de la Constitución española, entiende que significa dos cosas diferentes: igualdad en el trato dado por la ley (o igualdad en la ley) e igualdad en la aplicación de la ley; la primera se corresponde con el que he llamado «sentido estricto» de la igualdad ante la ley, y la segunda, con el «sentido amplio» de la misma (cf. STC 49/1982, FJ $2^{\circ}$ y STC 144/1988, FJ $1^{\circ}$ ); véase también esta distinción en Sadurski, 1986 y Ruiz Miguel 1995, 111-116. 
mún. Una vez definido lo que merecen (por ejemplo, determinados derechos), no hay razón alguna para atribuírselo a unos y negárselo a otros. Creo que a partir de aquí puede cuestionarse la distinción tradicional entre derechos de libertad y derechos de igualdad, puesto que éstos también son derechos de libertad ${ }^{17}$. Cuando se dice de los derechos sociales que son derechos de igualdad, o que sirven a la igualdad, creo que lo que se quiere decir es que sirven a la igualación de la libertad, aunque, para asumir esta afirmación, quizá haya que aceptar el uso de la categoría de la libertad «real» (recuérdese lo señalado más arriba). La libertad de expresión, la libertad de residencia o de circulación, etc., hacen a las personas más libres; pero el derecho a la educación, a la seguridad social, a la asistencia sanitaria, también. Desde este punto de vista, no veo qué diferencia hay entre unos y otros: si acaso, que el segundo tipo de derechos beneficia más a unas personas que a otras, mientras que el primer tipo de derechos beneficia a todas por igual, pero eso sucede sólo porque existen normas jurídicas anteriores que generan discriminación en el disfrute de la libertad (por ejemplo, las que protegen la propiedad privada). En una sociedad desigualitaria, es lógico que unos derechos sirvan a todos y otros, sólo a algunos; pero una sociedad desigualitaria es normativamente incompatible con una teoría coherente de los derechos humanos ${ }^{18}$.

En resumen, creo que sería interesante reconsiderar si es necesario establecer un derecho a la igualdad además del derecho a la libertad, dado que, asumidos los presupuestos de una teoría de los derechos humanos, ese derecho a la libertad tiene que atribuirse a todos los seres humanos por igual; y cuando se afirma el derecho a la igualdad, en realidad no se dice nada más que eso ${ }^{19}$. Históricamente, la afirmación del derecho a la igualdad ha servido para oponerla a una concepción elitista o clasista de la libertad, dominante durante algún tiempo en la filosofía política liberal, y vinculada con la defensa de los intereses de la burguesía; pero si lo que se quiere es generalizar el disfrute de la libertad, me parece mejor camino modificar la defi-

${ }_{17}$ Véase recientemente Gargarella 1998, donde se ofrecen argumentos para cuestionar la distinción entre derechos individuales y derechos sociales; también Prieto 1998, 77-78.

${ }^{18}$ Llamo «sociedad desigualitaria» a una sociedad donde los recursos y las oportunidades vitales están desigualmente repartidas, siempre que esta desigualdad no sea consecuencia de la aplicación de un criterio éticamente justificado. Una forma parecida de enfocar la relación entre derechos sociales, libertad e igualdad aparece en De Asís, 2000, 153.

${ }^{19}$ Liborio Hierro escribe: «nuestro ideal de igualdad es la igualdad entre todos los seres humanos en los recursos adecuados para satisfacer las necesidades básicas, de forma que permitan a todos y cada uno desarrollar de forma equiparablemente autónoma su propio plan de vida». Insisto en que, para aceptar esta afirmación, no hace falta recurrir al derecho a la igualdad: basta con recordar cuál es el fundamento de la teoría de los derechos humanos propuesto por el propio Hierro: la igual autonomía moral de todos los seres humanos. 
nición de la libertad que oponerle el valor de la igualdad. De hecho, tratar de plantear ciertos problemas jurídicos o políticos en términos de conflicto entre la libertad y la igualdad suele llevar a callejones sin salida lógica y suele servir para justificar intereses ilegítimos, como el de favorecer la libertad de unos más que la de otros (en el abstracto nombre de la libertad) o el de perjudicar la libertad de todos (en el abstracto nombre de la igualdad).

\section{El derecho a la seguridad}

Liborio Hierro distingue dos posibles formas de la seguridad: la seguridad jurídica y la seguridad frente al riesgo, y rechaza ésta como candidata a constituir un derecho porque considera que pertenece, más bien, al derecho a la igualdad (o al derecho a la libertad, si se acepta mi razonamiento de párrafos anteriores), de manera que interpreta la seguridad como seguridad jurídica y define el derecho correspondiente con estos términos:

Todo ser humano tiene derecho a la protección normativa de su vida, su integridad física y moral y su libertad de acción frente a la acción ajena y a que los límites de su libertad estén establecidos por reglas generales y claras, preeestablecidas, públicas, irrectroactivas y eficaces.

A mi juicio, la seguridad jurídica no debe ser considerada como un derecho humano ni tampoco como un derecho subjetivo de otro tipo sino que, más bien, debe entenderse como «eficacia de un sistema jurídico en tanto sistema normativo». Hablando en general, la seguridad debe considerarse como el resultado que se obtiene al aplicar determinadas técnicas (generalmente normativas, aunque no sólo) a diversos aspectos de la vida de las personas. Si tenemos en cuenta el Derecho en particular (una particular técnica social normativa), de él se predica seguridad (seguridad jurídica) porque se trata de una técnica que obtiene un cierto grado de previsibilidad de los comportamientos humanos; pero eso sólo significa que el Derecho es técnicamente apropiado para la consecución de determinados fines (la previsibilidad de la conducta; de la misma forma puede decirse que el conocimiento genera seguridad porque torna previsibles acontecimientos que no lo serían si no fueran objeto del mismo) ${ }^{20}$. Dicho esto, creo que la seguridad jurídica no conlleva una carga axiológica propia, sino que ésta descansa en aquellos bienes que están asegurados por el uso de la técnica del Derecho. Cosa distinta es defender la bondad ética del uso del Derecho si éste sirve para garantizar un determinado nivel de seguridad en el disfrute de dichos bienes, pero aquí se predica la bondad ética de aquéllos que recurren al uso

${ }^{20}$ Estoy suponiendo que, dada la conexión necesaria entre técnica normativa y seguridad, no es posible imaginar un sistema jurídico que no genere seguridad. Sí es posible, en cambio, que los distintos sistemas jurídicos la generen en distinta medida, y es correcto calificar como técnicamente mejor al que más genera. 
de la técnica apropiada para fines éticos, y no la bondad ética de la técnica jurídica en sí, dado que ésta podría servir igualmente a fines éticamente in$\operatorname{correctos}^{21}$. Por supuesto, lo que aquí está en juego es el debate sobre el valor moral intrínseco del Derecho, pero no es éste el lugar oportuno para desarrollarlo. A mi juicio, los derechos derivados del derecho a la seguridad pueden configurarse sin problemas como derechos derivados del derecho a la libertad, puesto que su función es precisamente asegurar modalidades de la libertad y no cualquier otra cosa.

Obsérvese también que la forma de definir el derecho a la seguridad es problemática desde un punto de vista lógico por dos razones, ambas relativas al hecho de que la idea de seguridad no puede situarse en el plano de los valores o derechos a proteger por el ordenamiento jurídico. La definición dice dos cosas: en primer lugar, que «todo ser humano tiene derecho a la protección normativa de su vida, su integridad física y moral y su libertad de acción»; pues bien, la protección normativa de la vida, la integridad física y moral y la libertad de acción puede traducirse como «el derecho a la vida, a la integridad física y moral y a la libertad de acción», luego lo que está diciendo el primer renglón de la definición es: «todo ser humano tiene derecho al derecho a...»; siguiendo el mismo criterio, podríamos estipular otro derecho, así definido: «todo ser humano tiene derecho al derecho al derecho a...» (derecho que podríamos denominar «derecho a la seguridad de la seguridad»), y así hasta el infinito; pero no veo cuál es el sentido de postular estos, por así decir, «derechos de segundo grado» que se pueden extender indefinidamente. Si una persona tiene un derecho, lo que se está diciendo precisamente es que tiene derecho a que se establezcan las normas oportunas para proteger un determinado bien. Si se acepta esto, creo que postular un derecho a la seguridad es redundante e innecesario. Por otra parte, no veo por qué el derecho a la protección normativa alcanza sólo a la vida, la integridad física y moral y la libertad de acción, y no también a otros bienes valiosos, como la educación, la salud, la vivienda, etc. (salvo que se entiendan incluidos en esos tres). En segundo lugar, la definición dice: «todo ser humano tiene derecho a que los límites de su libertad estén establecidos por reglas generales y claras, preestablecidas, públicas, irrectroactivas y eficaces»; mi opinión es que si los límites de la libertad estuvieran establecidos de otro modo, simplemente no estarían establecidos y, en consecuencia, la propia libertad no quedaría establecida. Por tanto, lo que el segundo renglón dice es: «todo ser humano tiene derecho a que su libertad quede establecida (normativamente)», lo cual parece innecesario, en función del argumento propuesto en este mismo párrafo.

\footnotetext{
${ }^{21}$ Esta última tesis es discutida por muchos, señaladamente por Fuller (1969).
} 
Sería interesante describir el proceso mediante el cual la seguridad jurídica ha pasado a ser considerada como un valor moral por parte de la tradición jurídica occidental, pero me limitaré a señalar que en este proceso es clave la necesidad de encontrar un expediente objetivo que justificase la obligación de obedecer el Derecho en los tiempos de auge del relativismo moral. Entiendo que la obligación de obedecer el Derecho nunca puede justificarse con base en la seguridad jurídica sino en los valores materiales que el Derecho efectivamente protege. Insistir en este punto permite denunciar el habitual recurso a la misma para tratar de fundar (incorrectamente) una obligación moral de obediencia al Derecho ${ }^{22}$.

\section{Observaciones finales}

El carácter tentativo de las acotaciones anteriores no permite hablar de conclusiones, pero añadiré dos observaciones más para terminar. Primero, quiero recordar que el trabajo de Liborio Hierro me parece excelente y lleno de aciertos, aunque yo me haya dedicado a comentar algunos de sus aspectos que me parecen problemáticos. Entre sus aciertos, yo destacaría: la forma de abordar la cuestión, ya comentada al principio; la defensa de la autonomía individual como fundamento único, necesario y suficiente de los derechos humanos; la conexión que establece entre la libertad positiva y negativa; la defensa de la igualdad de oportunidades como elemento necesario de una teoría de los derechos humanos; y la renuncia a incluir en dicha teoría al derecho de propiedad privada y a otros vinculados con él (herencia y libertad de empresa, por ejemplo).

Segundo, respecto de si existe un núcleo duro de derechos humanos, mi opinión es la siguiente: sí existe si lo entendemos como elemento esencial de todos y cada uno de los derechos humanos; no existe si lo entendemos como un grupo especialmente importante de derechos. Los derechos humanos forman un entramado único al servicio de la autodeterminación individual; cualquier pieza del entramado es necesaria para dicha autodeterminación, y sólo el conjunto es suficiente (a veces se habla de «indivisibilidad» de los derechos humanos para expresar esta idea). Si defendemos la mayor importancia de unos u otros derechos, estaremos favoreciendo la autodeterminación de unos individuos y perjudicando al tiempo la de otros, y ello no está autorizado por el presupuesto igualitario de toda teoría de los derechos humanos. La razón por la cual en distintos contextos espaciales y temporales se reclaman unos u otros derechos como más importantes radica en el hecho de que, en cada contexto, son derechos diferentes los que están en peligro o se echan a faltar.

\footnotetext{
22 Véase el argumento en González Vicén 1979, 376-385.
} 


\section{Bibliografía citada}

Aristóteles, 1985: Ética nicomáquea (Madrid, Gredos, trad. J. Pallí).

Aristóteles, 1989: Política (Madrid, Gredos, trad. J. Marías y M. Araujo).

N. Bobbio, 1993: Igualdad y libertad (Barcelona, Paidós, trad. P. Aragón Rincón).

R. Dworkin, 1984: Los derechos en serio (Barcelona, Ariel; trad. M. Guastavino).De Asís, 2000: «La igualdad en el discurso de los derechos», en Los derechos: entre la ética, el poder y el Derecho (edición de J. A. López García y J. A. del Real, Madrid, Dykinson), 149-168.

J. De Lucas, 1993: El concepto de solidaridad (México, Fontamara).

J. De Lucas, 1996: «La igualdad ante la ley», en El Derecho y la justicia (edición de E. Garzón Valdés y F. Laporta, Madrid, Trotta), 493-500.

R. Dworkin, 1984: Los derechos en serio (Barcelona, Ariel; trad. M. Guastavino).

E. Fernández, 1984: Teoría de la justicia y derechos humanos (Madrid, Debate).

L. L. Fuller, 1969: The Morality of Law (New Haven y Londres, Yale University Press).

R. Gargarella, 1998: «Primeros apuntes para una teoría de los derechos sociales. ¿Es posible justificar un tratamiento jurídico diferenciado para los derechos sociales e individuales?», en Jueces para la Democracia, 31, 11-15.

J. González Amuchastegui, 1991: «Notas para la elaboración de un concepto de solidaridad», en Sistema, 101.

F. González Vicén, 1979: Estudios de filosofía del Derecho (Universidad de La Laguna).

L. Hierro, 1998: Estado de Derecho: problemas actuales (México, Fontamara).

H. Kelsen, 1991: ¿Qué es justicia? (Barcelona, Ariel, trad. A. Calsamiglia).

F. Laporta, 1983: «Sobre el uso del término libertad en el lenguaje político», en Sistema, 52, 23-43.

F. Laporta, 1985: «El principio de igualdad: introducción a su análisis», en Sistema, 67, 3-31.

G. Peces-Barba, 1993: Derecho y derechos fundamentales (Madrid, Centro de Estudios Constitucionales).

G. Peces-Barba, 1995: Curso de derechos fundamentales (Madrid, BOE/Univ. Carlos III).

L. Prieto, 1994: «Nota sobre el concepto de derechos fundamentales», enJ. M. Sauca (ed.), Problemas actuales de los derechos fundamentales (edición de J. M. Saúca, Madrid, BOE), 181-189.

L. Prieto, 1998: Ley, principios, derechos (Madrid, Dykinson/Instituto de Derechos Humanos Bartolomé de las Casas).

G. Radbruch, 1974: Introducción a la filosofía del Derecho (México, Fondo de Cultura Económica, trad. W. Roces).

A. Ruiz Miguel, 1995: «Las huellas de la igualdad en la Constitución», en Pensar la igualdad y la diferencia. Una reflexión filosófica (edición de M. R. Mate, Madrid, Visor/Argentaria), 109-129.

A. Ruiz Miguel, 1997: «Equality before the Law and Precedent», en Ratio Juris, $10 / 4,372-391$. 
W. Sadurski, 1986: «Equality Before the Law: A Conceptual Analysis», en Australian Law Journal, 60, 131-138.

J. Rawls, 1985: Teoría de la justicia (México, Fondo de Cultura Económica, trad. M. D. González).

A. Ross, 1997: Sobre el Derecho y la justicia (Buenos Aires, Eudeba, trad. G. R. Carrió).

P. Singer, 1991: Ética práctica (Barcelona, Ariel, trad. M. Guastavino). 